\title{
Label-free tissue scanner for colorectal cancer screening
}

\author{
Mikhail E. Kandel \\ Shamira Sridharan \\ Jon Liang \\ Zelun Luo \\ Kevin Han \\ Virgilia Macias \\ Anish Shah \\ Roshan Patel \\ Krishnarao Tangella \\ Andre Kajdacsy-Balla \\ Grace Guzman \\ Gabriel Popescu
}




\title{
Label-free tissue scanner for colorectal cancer screening
}

\author{
Mikhail E. Kandel,,$^{\mathrm{a}, \mathrm{b}, \dagger}$ Shamira Sridharan, ${ }^{\mathrm{a}, \mathrm{c}, \mathrm{d}, \mathrm{\dagger}}$ Jon Liang, ${ }^{\mathrm{a}}$ Zelun Luo, ${ }^{\mathrm{a}}$ Kevin Han, ${ }^{\mathrm{a}}$ Virgilia Macias, ${ }^{\mathrm{e}}$ \\ Anish Shah, ${ }^{\mathrm{e}}$ Roshan Patel, ${ }^{\mathrm{e}}$ Krishnarao Tangella, ${ }^{\mathrm{f}}$ Andre Kajdacsy-Balla, ${ }^{\mathrm{e}}$ Grace Guzman, ${ }^{\mathrm{e}}$ and \\ Gabriel Popescu ${ }^{\mathrm{a}, \mathrm{b}, \mathrm{c}, \boldsymbol{*}}$ \\ aUniversity of Illinois at Urbana-Champaign, Beckman Institute of Advanced Science and Technology, Quantitative Light Imaging Laboratory, \\ Urbana, Illinois, United States \\ bUniversity of Illinois at Urbana-Champaign, Department of Electrical and Computer Engineering, Urbana, Illinois, United States \\ 'University of Illinois at Urbana-Champaign, Department of Bioengineering, Urbana, Illinois, United States \\ dUniversity of California, Biomedical Engineering Department, Davis, California, United States \\ eUniversity of Illinois at Chicago, Department of Pathology, Chicago, Illinois, United States \\ ${ }^{\mathrm{f} C}$ Christie Clinic, Department of Pathology, Urbana, Illinois, United States
}

\begin{abstract}
The current practice of surgical pathology relies on external contrast agents to reveal tissue architecture, which is then qualitatively examined by a trained pathologist. The diagnosis is based on the comparison with standardized empirical, qualitative assessments of limited objectivity. We propose an approach to pathology based on interferometric imaging of "unstained" biopsies, which provides unique capabilities for quantitative diagnosis and automation. We developed a label-free tissue scanner based on "quantitative phase imaging," which maps out optical path length at each point in the field of view and, thus, yields images that are sensitive to the "nanoscale" tissue architecture. Unlike analysis of stained tissue, which is qualitative in nature and affected by color balance, staining strength and imaging conditions, optical path length measurements are intrinsically quantitative, i.e., images can be compared across different instruments and clinical sites. These critical features allow us to automate the diagnosis process. We paired our interferometric optical system with highly parallelized, dedicated software algorithms for data acquisition, allowing us to image at a throughput comparable to that of commercial tissue scanners while maintaining the nanoscale sensitivity to morphology. Based on the measured phase information, we implemented software tools for autofocusing during imaging, as well as image archiving and data access. To illustrate the potential of our technology for large volume pathology screening, we established an "intrinsic marker" for colorectal disease that detects tissue with dysplasia or colorectal cancer and flags specific areas for further examination, potentially improving the efficiency of existing pathology workflows. ๑2017 Society of Photo-Optical Instrumentation Engineers (SPIE) [DOI: 10.1117/1.JBO.22.6.066016]
\end{abstract}

Keywords: quantitative phase imaging; label-free; colorectal cancer; screening; slide scanner.

Paper 160874RR received Dec. 23, 2016; accepted for publication May 22, 2017; published online Jun. $24,2017$.

\section{Introduction}

Colorectal cancer develops from benign adenomatous polyps that advance to carcinoma through a series of genetic mutations over the course of 5 to $10 \mathrm{yr}^{1}$ Early diagnosis of colorectal cancer is strongly correlated with reduced disease-specific mortality. Cancers diagnosed at an early stage (localized) have an $89.8 \%$ 5-year survival rate compared with a $12.9 \%$ 5-year survival rate for patients with distant metastasis or late-stage disease. ${ }^{2}$ In United States, colonoscopy is the preferred form of screening, and the percentage of individuals in the age group of 50 to $75 \mathrm{yr}$ who underwent colorectal cancer screening increased from $54 \%$ to $65 \%$ from 2002 to $2010 .{ }^{3}$ The prevalence of adenoma among all individuals undergoing colonoscopy is $25 \%$ to $27 \%$, and the prevalence of high-grade dysplasia and colorectal cancer is even lower, $1 \%$ to $3.3 \% .^{4,5}$ However, a biopsy or polyp removal is performed in $50 \%$ of all colonoscopies as current screening methods cannot distinguish adenoma from a benign polyp with high accuracy. ${ }^{6}$ A pathologist examines all excised polyps to determine if the tissue is benign,

\footnotetext{
*Address all Correspondence to: Gabriel Popescu, E-mail: gpopescu@illinois .edu
}

†The authors contributed equally to this work. dysplastic, or cancerous to determine if further treatment is necessary.

The Patient Protection and Affordable Care Act in the United States now requires all insurances to cover cancer screening strategies recommended by the United States Preventive Services Task Force, ${ }^{7}$ and recent studies have shown the critical need for colorectal cancer screening in Asia in light of an alarming increase in the prevalence of this disease. ${ }^{8-10}$ Thus, it is very likely that the number of colon cancer screening cases examined by pathologists will increase. Considering the small proportion of cases with advanced neoplasms in the midst of a large volume of tissue excised during screening, a quantitative tool that can flag dysplastic or cancerous tissue with a high level of accuracy will be invaluable for early colorectal cancer detection.

New technologies that can perform automated tissue investigation and thus reduce the dependence on manual examination are imperative for efficient large-scale screening strategies. Already, widespread implementation of the Papanicolaou test (pap smear) for cervical cancer screening benefited from semiautomated computational tools. The FDA-approved BD Focal Point Slide Profiler ${ }^{\mathrm{TM}}$ reduces pathology caseloads by $25 \%$ by eliminating normal cases, forwarding the remaining $75 \%$ of

$1083-3668 / 2017 / \$ 25.00$ @ 2017 SPIE 
cases to the pathologist for a manual examination with a risk group designation. ${ }^{11}$ Critical to the proper operation of such systems is the staining procedure, which is designed to match narrow calibration thresholds. The details of these procedures are proprietary and require continuous quality review throughout the machine's operating lifetime. ${ }^{13}$

Label-free scattering and imaging techniques have been developed recently to perform tissue diagnosis based on the intrinsic tissue architecture modifications associated with cancer onset and development (see, e.g., Refs. 14-25). As our work focused on rapid and high-resolution imaging of thin samples, we choose to use quantitative phase imaging (QPI). ${ }^{26} \mathrm{QPI}$ is an emerging field that shows promise for quantitative assessment of cell and tissue properties, including cell growth, ${ }^{27-29}$ cellular physiology, ${ }^{30-33}$ single-cell tomography, ${ }^{34-39}$ and hematology, ${ }^{40-43}$ as well as cancer diagnosis. ${ }^{19,44-47}$ In essence, the advantage of a label-free approach is that variables introduced by staining and color balance disappear, yielding information well suited for computer analysis, and QPI is one of the most sensitive techniques in this class of imaging modalities.

Here, we present, for the first time to our knowledge, a QPIbased tissue scanner capable of acquisition rates that are comparable with the existing commercial, intensity-based scanners. Our label-free scanner combines optical hardware as well as dedicated, highly parallelized software algorithms for data acquisition. We apply this technology, for the first time to our knowledge, for performance of automatic colon tissue screening, using intrinsic, "self-normalizing," tissue markers, showing how such a system can be used to remove normal (benign) cases from manual investigation by pathologists.
Importantly, such a measurement does not require staining or calibration. Therefore, unlike with staining, signatures developed from phase information can be shared across laboratories and instruments without modification.

\section{Materials and Methods}

\subsection{Study Design}

In this work, we imaged a tissue microarray (TMA) set containing tissue with colon carcinoma, intramucosal carcinoma, dysplasia, hyperplasia, and normal colonic mucosa. Quantitative features were extracted from the image to construct a support vector machine (SVM) classifier using MATLAB ${ }^{\circledR}$ to flag cases with dysplasia, intramucosal carcinoma, and carcinoma as high index of suspicion using $25 \%$ of the cases in the TMA set. The classifier was then tested on the remaining $75 \%$ cases in the TMA set as an internal validation. We envision that this screening process will reduce the workload for pathologists. To demonstrate the application of our technique to biopsies, we imaged a large section of surgically resected tissue using a second instrument. The SVM classifier was then tested on the surgical specimen as an external validation.

\subsection{Interferometric Microscopy}

We direct the light from a conventional phase-contrast microscope (Axio Observer Z1, Zeiss) onto the spatial light interference microscopy (SLIM) module [Cell Vista SLIM Pro, Phi Optics, Inc., Fig. 1(a)]. The module consists of lenses, polarization control, and a fast liquid crystal phase modulator (a)

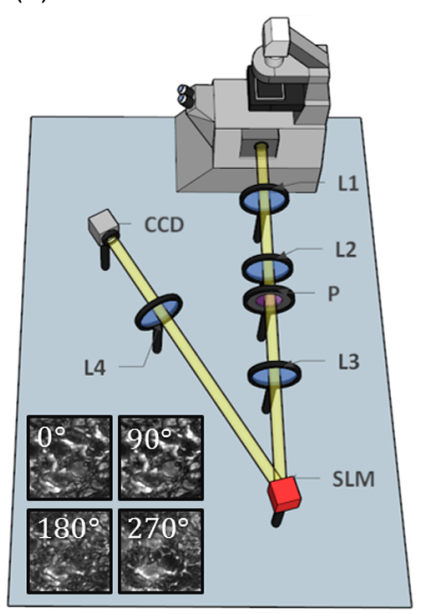

(c)

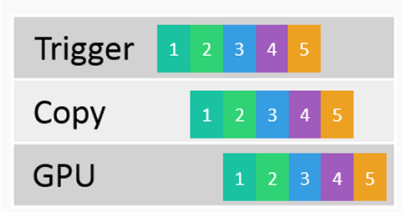

(b)

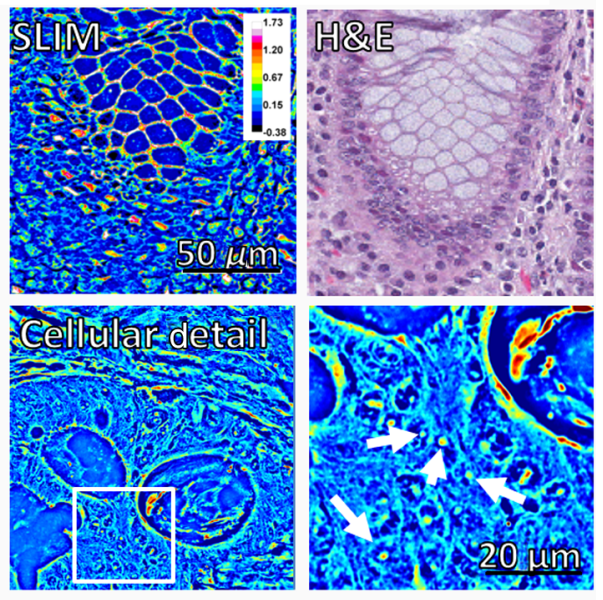

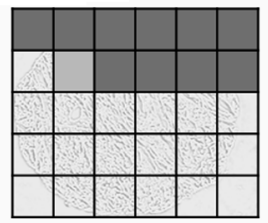

Fig. 1 High-throughput imaging system enables label-free cancer diagnosis. (a) SLIM is a white-light interferometry technique that attaches onto an existing phase-contrast microscope. The phase offset between the scattered and transmitted light is manipulated by an SLM. (b) A home-built C++ code overlaps camera acquisition, data copy, computation, and mechanical motion to maximize hardware parallelism. Previous frames are processed while the current one is acquired. (c) The resulting SLIM images have sufficient intrinsic contrast to match or outperform conventional staining procedures for colorectal cancer screening. Cellular level detail, such as nucleoli (white arrows), is clearly visible. 
( $\sim 10$-ms stabilization time) operating in reflection mode. The modulator is located at the Fourier plane of the image field and controls the offset between scattered and transmitted light, effectively adjusting the phase shift associated with the ring found in the phase-contrast objective. Following the interferometry technique described in Ref. 48, the images are collated to produce a quantitative phase image, with values at each pixel in units of radians. All images presented here were obtained using a $40 \times$ $/ 0.75$ NA objective, which yields a $330 \times 330 \mu \mathrm{m}^{2}$ field of view, sampled by a camera with five megapixels (Zyla, Andor).

Initially, achieving a flat field required that the system be carefully aligned for each objective. In a later revision of the optical assembly (not used here), the field of view is dynamically adjusted with a manual "zoom lens" (Phi Optics proprietary, similar to Thorlabs PN: SM1NR1) positioned between the microscope and the SLIM add-on module.

\subsection{Software Architecture}

Our software overlaps computation with necessary hardware events, such as spatial light modulator (SLM) modulation and camera transfer time. To achieve real-time performance [Fig. 2(a)], we use three decoupled threads in a pipeline fashion. The first thread is responsible for triggering the acquisition of new images and controlling the SLM. A second thread receives and transfers new images to the graphics processing unit (GPU), while a third thread renders the phase images and writes them to the disk [Fig. 2(b)]. This "producer-consumer" model is necessary as scientific cameras have precise shutter open triggers, but the availability of acquired data depends on the computer load. For example, a camera that is triggered every $10 \mathrm{~ms}$ has data arriving in $12 \mathrm{~ms} \pm 2 \mathrm{~ms}$ intervals. Without this scheme, the throughput would be $14 \mathrm{~ms}$ rather than $10 \mathrm{~ms}$, and camera aperture jitter would perturb time-critical experiments. We found that using Windows Multimedia Timers, along with disabling latency-prone hardware devices further reduces aperture jitter. As most of the computation is carried out on the GPU, the Windows Task Manager rarely displays appreciable central processing unit (CPU) utilization. Thus, by separating acquisition and file writing, the program implicitly handles unpredictable write speeds, such as the latencies due to disk seeks on large redundant array of independent disks arrays. Even solid-state drives benefit from this approach as their speed is hard to know a priori (due to the so-called "solid state drive write cliff"). ${ }^{49}$

To avoid redundant memory copies, a large buffer is acquired at program initialization and pointers rather than deep copies are used to exchange data between threads. Further, these pointers are used to detect acquisition failure by verifying the order and arrival of triggered frames. When an error occurs, the acquisition event is repeated, although some categories of errors require the camera to be software reset. In these cases, the necessary synchronization is handled by a record keeping structure. In this structure, frames are pooled into three categories: "free," "inside camera," and "inside write queue." In the event of an error, a lock is held until the necessary corrections are made, resetting the camera or waiting for more files to be written to the disk, implicitly pausing the acquisition threads.

To facilitate scanning slides and other forms of programmed automation, "capture dialogs" generate a list of steps outlining the precise state of the microscope [Figs. 3(a) and 3(b)]. This list is then processed [Fig. 3(c), "schedule"] with one thread dedicated to triggering the camera, shifting the SLM, and moving the microscope stage. A second thread is responsible for (a)

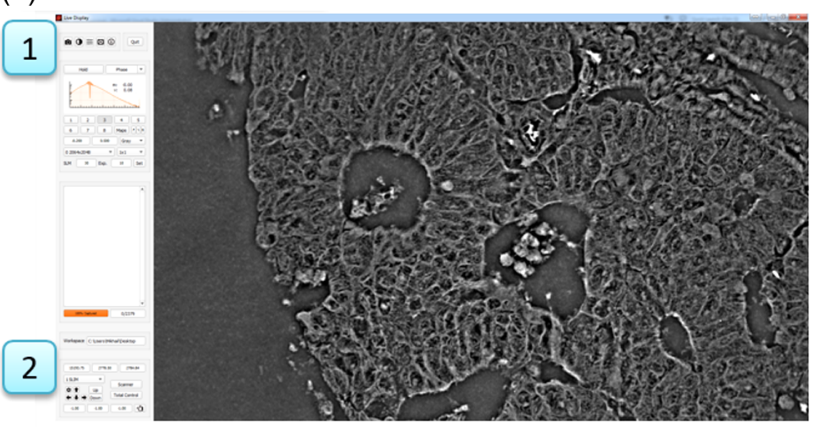

(b)

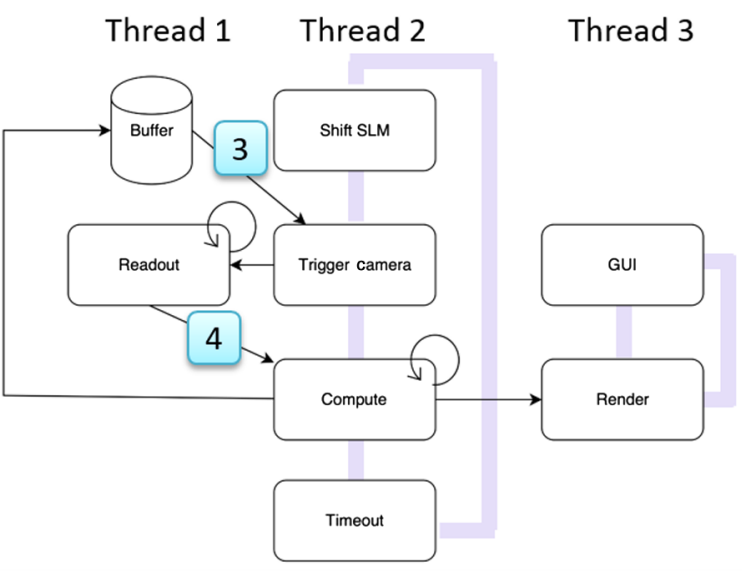

(c)

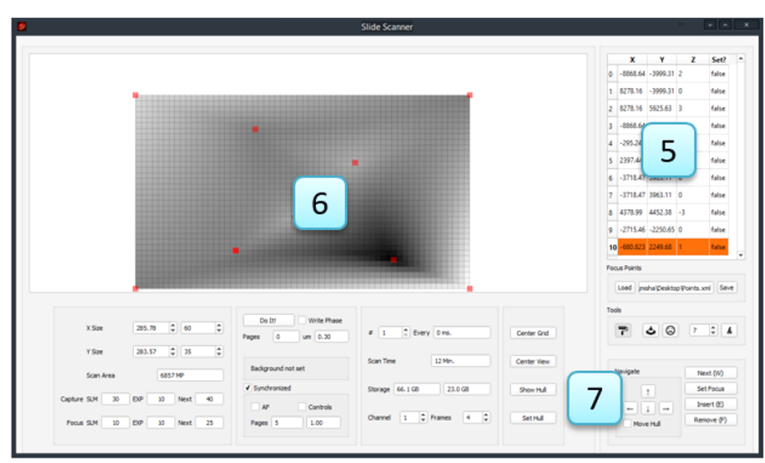

Fig. 2 User-friendly QPI slide scanning interface enables rapid digitization of microscope slides: (a) live image, (b) phase retrival, and (c) scanner dialog. The live interface enables the SLIM imaging process in real time (inset 1). Here, SLIM imaging is seamlessly integrated among other channels of the microscope. The SLIM channel can be selected or mixed with the fluorescence, dark-field, differential interference contrast, and phase-contrast channels that operate on the existing microscope (inset 2). To account for mismatch between consumer and producer threads, the "triggering" process (inset 3) releases a single frame while the readout process is capable of receiving multiple frames (inset 4). In the acquisition dialog, the scanner surface (inset 5) is represented as a rectangle with shading indicating the height variation due to manually selected focus points (inset 6). Various settings, such as the depth of the autofocus system, can be configured through the interface (inset 7).

receiving the images, computing autofocus parameters, and record keeping to redo frames in the case of acquisition failure. Finally, a third thread is responsible for writing files to the disk. With tightly overlapped software design, the rate limiting factors in our data acquisition procedure are hardware parameters, such as XY stage speed, SLM stability, and camera readout time.

Because of this parallelization procedure, we are able to sustain 155.5 megapixel SLIM phase maps per second. 
(a)

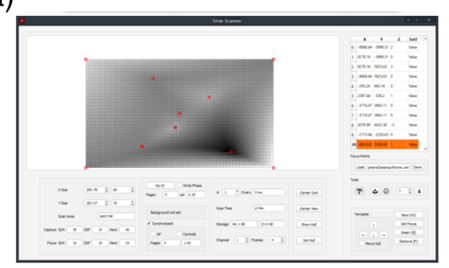

(c) (b)

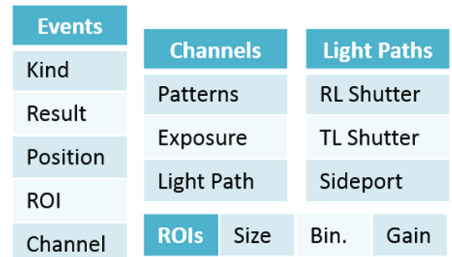

(d)

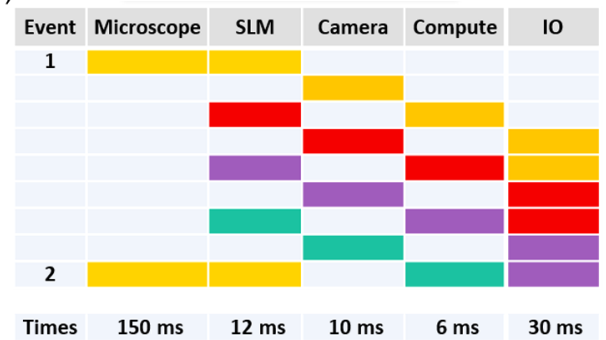

Fig. 3 Software architecture for high-throughput imaging with error correction. The software architecture was designed to maximize computational overlap while providing deterministic operation for error correction. (a) Capture dialog, showing the slide scanning and "multi-ROl" acquisition modes, generates a list of events and channel settings. (b) The event list is then processed by a scheme where one thread is assigned to triggering (moves stage, shifts SLM, and shutter open on camera) and a second thread receives images and performs bookkeeping for error detection (busy wait on new images and compute focus parameters). (c) Schedule of events. Acquired images are pushed onto a file writing thread, which enables the software to briefly exceed hard disk write speeds without affecting the acquisition. To avoid redundant data copy, along with system overhead during high-speed acquisitions, a large memory buffer is preallocated and pointers are used in place of whole images. (d) The validity of the pointers is used for error detection.

\subsection{Phase-Based Autofocusing Procedure}

To achieve optimal image quality, our slide scanner adjusts the focus at each mosaic tile. This procedure benefits from knowledge of the approximate plane of best focus, and, thus, autofocusing is performed offset from a user-defined focus plan [Fig. 4 (a)]. To enable dynamic adjustment of the plane of best focus as the scan progresses, the tile positions and associated focus points are stored as a Delaunay triangulation. ${ }^{50}$ Nevertheless, during the course of this work, we found that dynamically adjusting the focus resulted in poor image quality, especially when our scanning procedure encountered large open areas or sharp discontinuities (residual paraffin). Therefore, we choose to keep this offset plane constant for the duration of the acquisition, and the depth scanning autofocus procedure is performed from this offset.

The autofocus algorithm ${ }^{51}$ proceeds by scanning through the $z$-depth and measuring the focus at each plane [Fig. 4(b)]. Iterative techniques, such as golden-section search, ${ }^{52}$ showed promise but were found to be significantly slower as the mechanized focus needed to traverse a larger distance. Further, anything other than a linear scan necessarily requires serializing the computation (waiting for pixel readout), effectively doubling the time needed per $z$-slice. Importantly, using a convex optimization technique is fundamentally unreliable because noise renders the focus curve nonmonotonic. Unlike a linear search, a misstep at the first bifurcation is irreversible and leads to a drastic deviation from the optimal result. Thus, our implementation samples along the $z$-axis at approximately five frames per second. The acquisition time is evenly divided between the fourframe phase shifting process and adjusting the piezo focus. After sampling the focus function, the algorithm performs a parabolic interpolation to choose the final image and, as the focus curve is smooth, interpolation helps to achieve a good result with few samples [Fig. 4(b), black circle].

Since an out-of-focus position generates a convolution with a Fresnel kernel, which smooths the edges of the phase map, the focusing metric relies on maximizing the variance of the twodimensional wavelet decomposition [Fig. 4(c)]. Specifically, we used the Haar wavelets that are a basis of edges, which are well suited to describing transient discontinuities.

The robustness of this metric can be assessed by its performance in cases where the plane of best focus is ambiguous. Such a case frequently occurs in live-cell imaging where there is a tendency for individual cells to round up (bulge) away from the glass, often during mitosis. Similar defects manifest themselves as bubbles of mounting medium or residual paraffin in imperfect tissue sections. The membrane circumscribing the cells appears as a sharp discontinuity and contributes disproportionately to the overall image variance, resulting in a counterintuitive best focus where the rest of the cells are blurred [Fig. 4(e)]. This further speaks to the difficulty of using an adaptive sampling technique (see the previous paragraph) because the "in-focus" image is expected to be globally optimal rather than locally optimal. As shown, using a metric that combines both the high- and low-frequency wavelet details image, the "fusion" scheme selects the desired result. Thus, owing to the stability of the common-path interferometer and the precision of the piezo focus, even relatively large focus steps can be used to localize the focus with high repeatability [Fig. 4(d)].

\subsection{Scanning and Image Assembly}

Mosaic tiles are acquired by translating an XY stage (Zeiss MCU2008), with an expectation of rigid misalignment along either the $X, Y$, or both. Hence, we opt to maximize the 
(a)

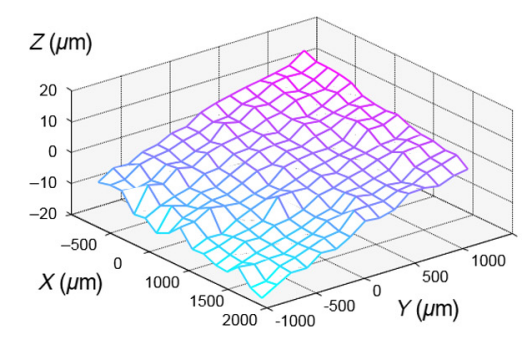

(c)

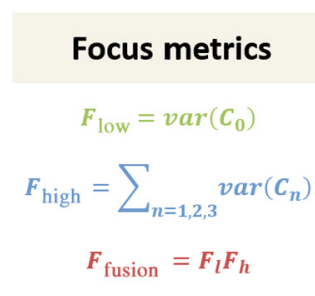

(e)

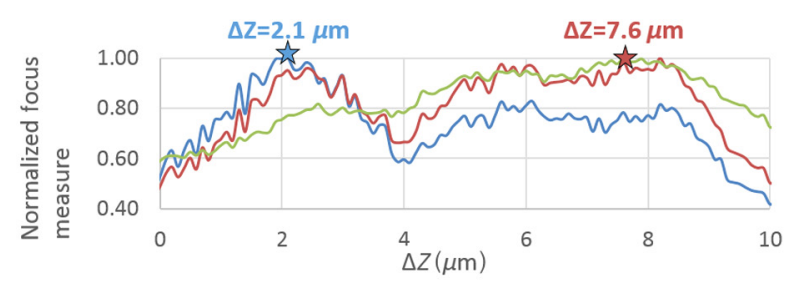

(b)

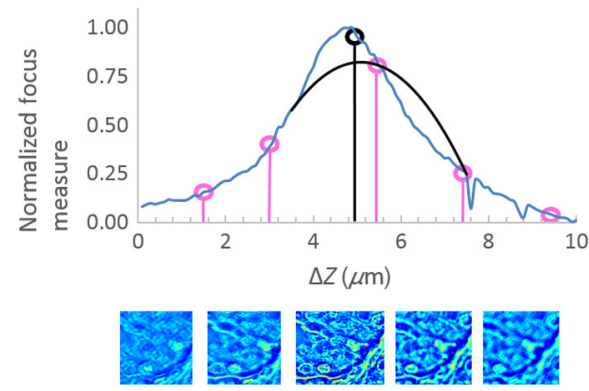

(d)

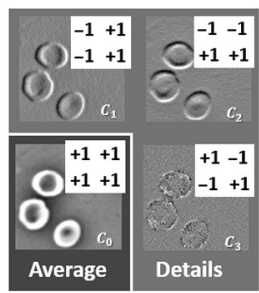

Fig. 4 Fusion of focus metrics resolves ambiguous cases. (a) Tilt correction, (b) focus optimization, and (c) a focus metric can be calculated from the variance of the wavelet decomposition details images obtained from the SLIM image. Such a scheme is useful for choosing a plane of best focus as there may exist local solutions for the plane of best focus. (d) To ascertain repeatability, the focus position at a different ROI (not shown) was taken 20 times, with a 7-point scan at a varying step size. We attribute the high level of repeatability (less than $50 \mathrm{~nm}$ ) to the interferometric stability of the SLIM image. (e) The weighting of low and high detail images focuses the image on the bulk of the sample rather than on high variance outliers. This allows for a nonambiguous solution to the plane of focus.

similarity between overlapping mosaic tiles using phase correlation $^{53}$ to perform image registration. To avoid memory limitations, our scheme consists of two passes on the data, with the first pass finding the optimal overlap and the second pass generating tiles for archival storage. The alignment procedure is designed to mask computation with disk access, with one thread responsible for loading data into a large buffer and a second thread performing the phase correlation procedure. Further, all image reads and the associated computations are stored in a cache, so subsequent use does not require further disk access. When the RAM is exceeded, the oldest image is removed from the cache. An additional performance improvement comes from ordering the pairs to minimize the potential number of times items can be removed from the cache. The required real-to-complex discrete Fourier transforms are performed on the GPU, with the computational portion occupying significantly less time than disk access. In most cases, this scheme avoids one of two of the Fourier transforms in the phase correlation algorithm.

Disagreement between estimates of the true tile position is resolved by a least-squares fit, producing a globally optimal configuration with a minimal alignment error. On the second pass, mosaic tiles are rendered according to this configuration, and the resulting images are generated for web viewing $(256 \times$
256 pixels) or archival storage $(10,000 \times 10,000$ pixels, see Fig. 6). While overlapping regions between mosaic tiles could be merged to achieve a more aesthetically pleasing seam, to avoid affecting the quantitative image statistics (particularly, the variance), overlapping regions contain data from (only) a single mosaic slice. Finally, a background correction image is calculated by selecting a small percentage of the data whose phase values are near zero (empty space) and removing this background from each frame. Following the initial tile generation, successive levels combined to create the mipmaps ("image pyramids") typically used in online image viewers.

The code is written in Python with GPU portions using PyCUDA. The rate limiting step is disk reading and writing. In this work, we used CATMAID ${ }^{54}$ and Zoomify. The principle advantage of CATMAID is easy annotation, and Zoomify operates without a dedicated server. A representative colon TMA slide is shown in Fig. 5.

One of the chief objectives of our slide scanning instrument is digitally archiving TMAs. To segment cores, we proceed with a two-pass $k$-means ${ }^{55}$-derived scheme operating on a stitched, downsampled image. The first pass identifies constituent pixels as belonging to a core and determines the center of mass and cropping box, resulting in a series of unlabeled boxes around 

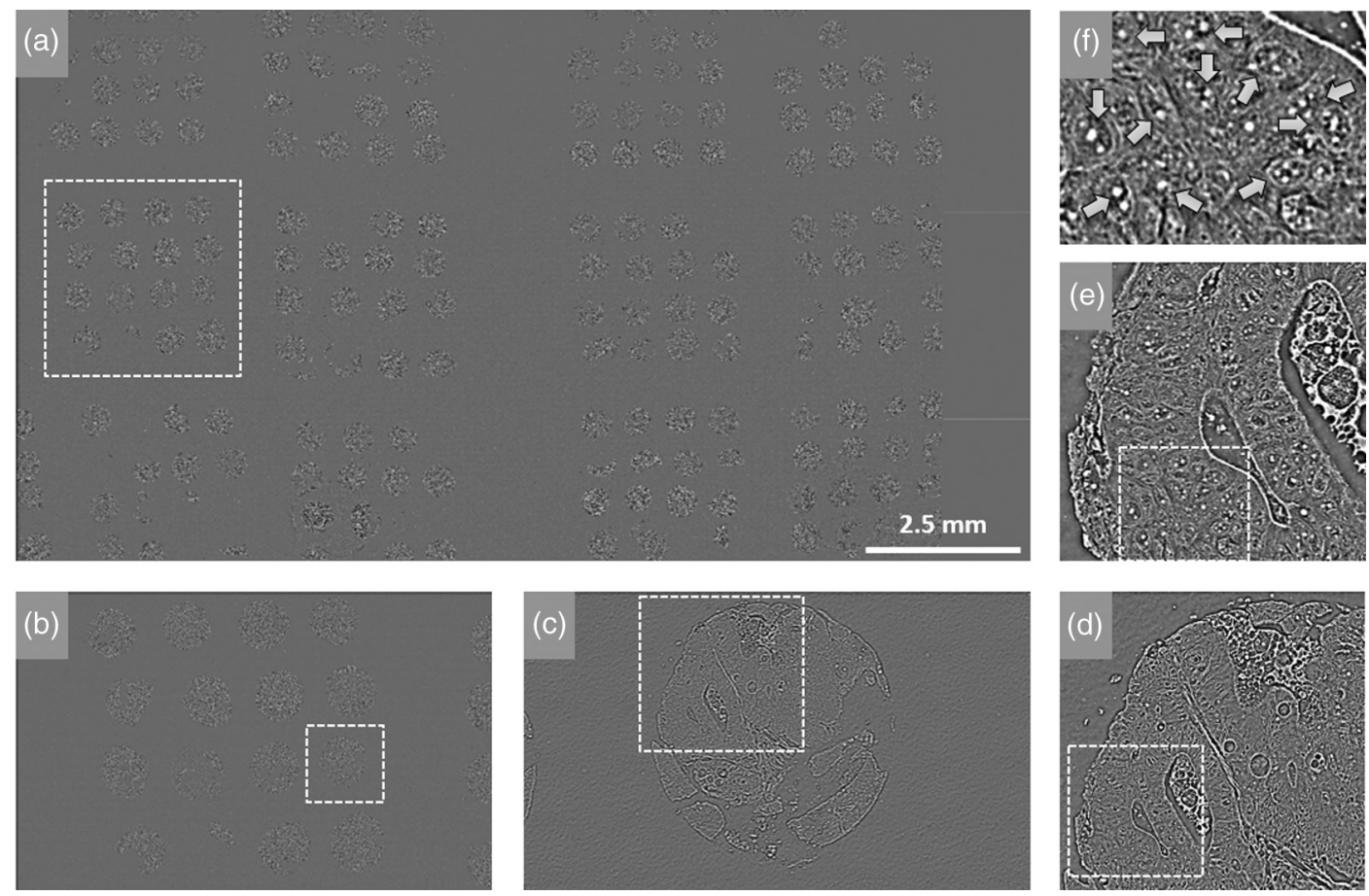

Fig. 5 Typical TMA slide. (a) Entire slide as seen on a web browser with virtually instantaneous loading. Original raw SLIM data amounts to $200 \mathrm{~Gb}$. (b)-(f) Google Maps TM type pyramid subsampling allows for a broad range of zoom levels to be accessed in real time remotely. Each image represents the region in the dash box shown in the previous image. Note that the TMA slide measures $1 \times 3$ inches, while our imaging was performed with submicron resolution, such that individual nucleoli (pointed by arrows in $\mathrm{f}$ ) are resolved. The TMA is available for remote browsing at http://light.beckman.illinois.edu/SI/Scanner/ Colon/Colon2/.

each core. Understanding that cores have irregular spacing, a further $k$-means algorithm is used to associate the centroids with their spacing on a regular grid. Essentially, a second pass of $k$-means is performed for each row and column to bin the centroids into the expected number of rows of columns. During the second pass, the $k$-sets for the clustering are known in advance, i.e., there are 100 cores along a $10 \times 10$ grid.

In the case of Fig. 11 (a biopsy), the process reduced $\sim 200$ gigabytes of floating-point phase maps into 2 gigabytes of web-ready 8-bit JPEGs.

\subsection{Tissue Microarray Cohort}

Our final goal is to apply our approach to biopsies. However, TMAs of colon resection specimens provide a controlled system, with a large number of distinct samples presented as 0.6 -mm disks on a single microscope slide. ${ }^{56}$ The morphological markers discovered in these TMA samples (gland solidity and phase median) are expected to be applicable to colonoscopic biopsies as well. The use of TMA obtained from colon resection specimens provides an ideal study material for addressing the morphological parameters of colon cancer progression. Furthermore, the use of TMAs is superior to biopsy samples as biopsy samples are not guaranteed to yield a stepwise colonic lesion progression from normal, to hyperplasia, dysplasia, and carcinoma.

A TMA was prepared with archival pathology material collected from 131 patients who underwent colon resection for treatment of colon cancer at the University of Illinois at Chicago (UIC) from 1993 to 1999. For each case, $0.6 \mathrm{~mm}$ in diameter colon core duplicates of tissue corresponding to "tumor, normal mucosa, dysplastic mucosa, and hyperplastic mucosa" were retrieved. Tissue cores were transferred using an MTA-1 manual arrayer (Beecher Instruments, Inc.) into a high-density array composed of four blocks with primary colon cancer $(n=$ 127 patients) and mucosa of normal ( $n=131$ patients), dysplastic ( $n=33$ patients), and hyperplastic colon ( $n=86$ patients). The gland median phase and solidity, computed as the ratio of the area of the gland to the area of the convex hull fitted around the gland, were used for classification. The tissue collection was performed in accordance with the procedures approved by the Institutional Review Board at UIC (IRB Protocol No. 2004-0317).

Two 4- $\mu \mathrm{m}$ sections were cut from each of the four blocks at UIC. The first section was deparaffinized and stained with hematoxylin and eosin (H\&E) and imaged using the Nanozoomer (bright-field slide scanner, Hamamatsu Corporation). One of the pathologists on our team made a diagnosis for all tissue cores in the TMA set, which was used as "ground truth" for analysis. A second adjacent section was prepared in a similar way but without the staining step. This slide was then sent to the Quantitative Light Imaging Laboratory at the University of Illinois at Urbana-Champaign for imaging. These studies followed the protocols outlined in the procedures approved by the Institutional Review Board at the University of Illinois at Urbana-Champaign (IRB Protocol No. 13900).

\subsection{Support Vector Machine Training}

Complete glands present on all cores were manually annotated on the SLIM images using a Wacom brand tablet and the region of interest (ROI) feature in ImageJ. Solidity, which is computed 
in ImageJ as the ratio of the area of the gland to the area of the convex hull fitted around the gland, and median phase value in each gland were measured. $25 \%$ of the cases in the TMA set were used to build an automated classifier for diagnosis. Solidity and phase median measurements were extracted from 454 glands from cores diagnosed as normal and 194 glands from cores diagnosed with cancer. These measurements were used to create a classifier with the SVM algorithm using MATLAB ${ }^{\circledR}$ (Fig. 10). A linear kernel was used to build the classifier, and sequential minimal optimization was used to calculate a hyperplane to separate the different classes of data. Evaluating a gland in the SVM yields values running on a continuum between "low index of suspicion," which would contain normal and hyperplastic tissue, and "high index of suspicion," which designates dysplastic and cancerous tissue.

\subsection{Internal Validation}

The TMA cores from the three slides, which were not used for building the classifier, were used for internal validation of the classifier. The solidity and phase median values are computed on $816,168,219$, and 704 glands from 100, 27, 34, and 102 cores diagnosed as normal, hyperplasia, dysplasia, and cancer, respectively. Using the SVM classifier, each gland was then stratified as "low" or "high" suspicion.

\subsection{External Validation}

A paraffin-embedded surgically resected colon sample was obtained from UIC. It was sectioned at $4-\mu \mathrm{m}$ thickness, deparaffinized, and later coverslipped with an aqueous mounting medium. The slide was imaged using the commercial SLIM system (Cell Vista SLIM Pro, Phi Optics, Inc.) with the tissue scanning software developed for this work. The slide was imaged with the $40 \times / 0.75 \mathrm{NA}$ objective and has a spatial resolution of $0.4 \mu \mathrm{m}$. The size of each field of view was $221 \times 165 \mu \mathrm{m}$, corresponding to $1392 \times 1040$ pixels on the CCD camera. To image the surgically resected tissue (Fig. 11), 15,589 fields of view were mosaicked together, with $\sim 10 \%$ overlap on all sides. The images were stitched together using an image alignment and registration software developed for this work. The resulting large image file was then cropped into 176 images of $10,000 \times$ 10,000 pixels, corresponding to a $1587.3 \times 1587.3 \mu \mathrm{m}^{2}$ field of view. Manual segmentation of glands was performed on these cropped images, using ImageJ, to measure the solidity and median phase value. The SVM classifier was used to classify the glands as low or high index of suspicion. The individual glandular diagnoses were then thresholded at $50 \%$ to obtain a single flagging measure for each $10,000 \times 10,000$ pixel tissue region. Thus, multiple diagnoses are performed in a spatially resolved manner and in specific regions flagged for pathology assessment, as shown Fig. 11.

\subsection{Effects of Thickness on Quantitative Phase Imaging Images}

We performed imaging of two adjacent tissue slices cut at $4-\mu \mathrm{m}$ thickness and measured the phase median and glandular solidity, the parameters used for diagnosis, for three glands in each section. As seen in our analysis, there are not significant differences in either the median (paired $t$-test, $p=0.46$ ) or the solidity (paired $t$-test, $p=0.34$ ), demonstrating that our technique is robust (Fig. 6).

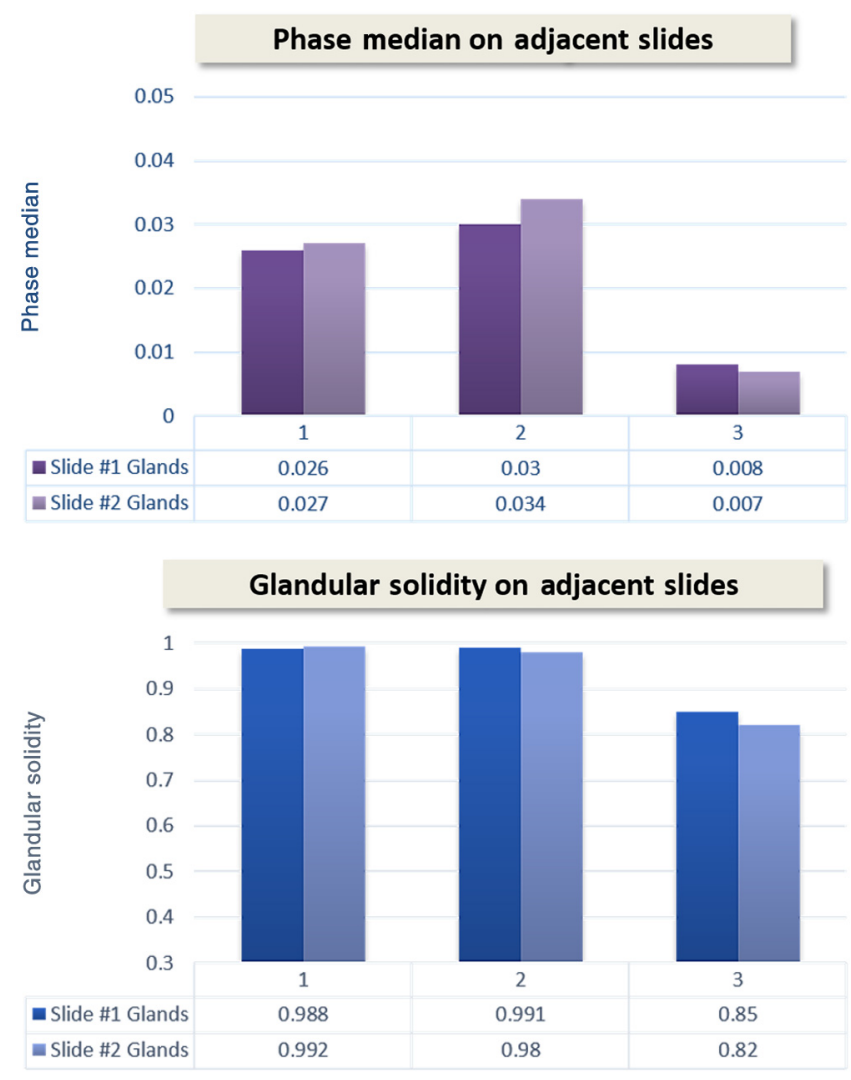

Fig. 6 Adjacent slices preserve diagnostic markers. We performed imaging of two adjacent tissue slices cut at 4- $\mu \mathrm{m}$ thickness and measured the phase median and glandular solidity, the two quantitative parameters used for diagnosis, for three glands in each section. As seen in our analysis, there are nonsignificant differences in both the median (paired $t$-test, $p=0.46$ ) and the solidity (paired $t$-test, $p=0.34)$.

This is in good agreement with Ref. 47, where measurements of slide thickness were performed using a scanning electron microscope (SEM). In that work, a paraffinized 3.5- $\mu \mathrm{m}$ thyroid tissue section was coated with gold/palladium before SEM measurements. Those images showed that the thickness of typical sample tissue is a continuum with a gradual decrease, followed by a gradual increase in thickness, indicating that changes in tissue morphology are primarily responsible for tissue thickness changes in microtome-derived histology slides.

\section{Results}

\subsection{Label-Free Tissue Scanner}

One of the chief outcomes of this work is a turnkey solution for a label-free pathology workflow. Figure 1(a) shows the SLIM module (Cell Vista SLIM Pro, Phi Optics, Inc.), which upgrades an existing phase-contrast microscope to produce quantitative phase maps. SLIM is described in detail elsewhere. ${ }^{39,48}$ In essence, SLIM operates by making the ring in the phase-contrast objective pupil "tunable." To achieve this, the image formed by a phase-contrast microscope is Fourier transformed at a plane of an SLM. At this plane, the image of the phase-contrast objective ring is perfectly matched to the mask on the SLM, and phase steps in increments of $\pi / 2$ are introduced sequentially. From four intensity images that correspond to different quarter-wavelength 
(a)

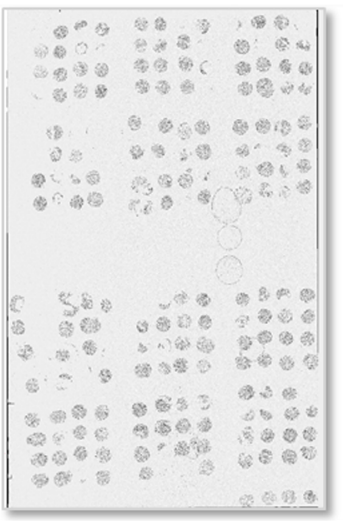

(c)

$\operatorname{argmin} \sum_{i=1}^{n} w_{i}\left|\Delta x_{i}\right|^{2}$

Least Squares

(e)

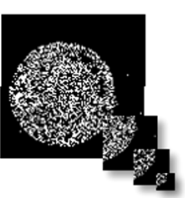

Background

Subtraction

Mipmapping

File Writing (b)

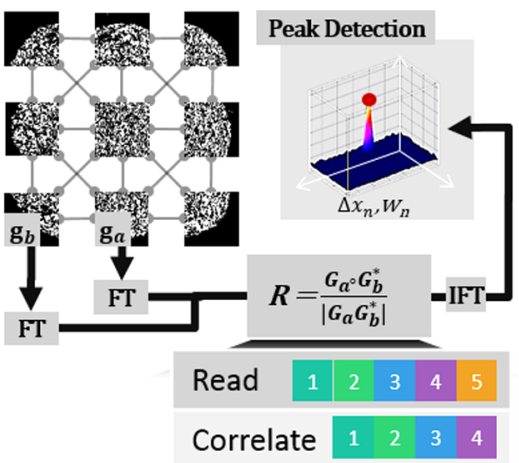

(d)

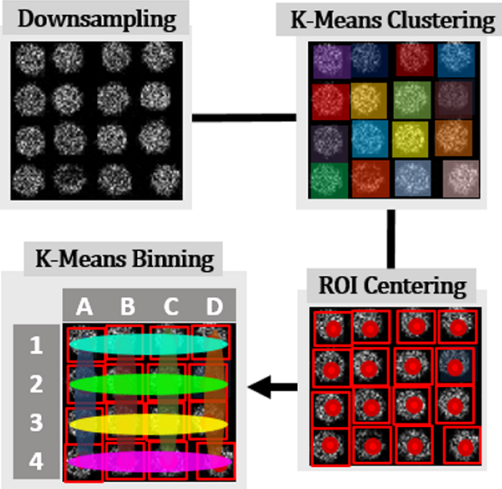

(f)

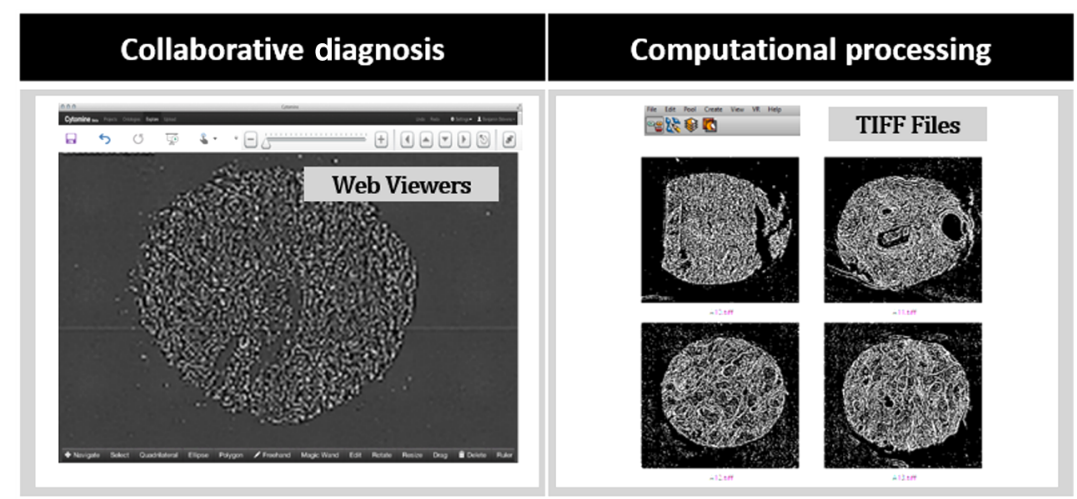

Fig. 7 Automated processing workflow for gigapixel SLIM images. (a) Unlabeled TMA, imaged by our SLIM tissue scanner. (b) Typical samples used in this paper result in tens of thousands of tiles. The tiles are assembled using a "phase correlation" scheme where the optimal displacement between neighboring tiles is determined by the location of the peak in the correlation image. In our implementation, disk access is overlapped with the correlation procedure. (c) Disagreements between estimated tile positions are resolved with a least-squares fit. (d) The result positions are used to generate image pyramids typically used for archival access. (e) Regularly spaced TMA cores are cropped and labeled by a thresholding technique. (f) Resulting images are ready for computational processing (files) and collaborative diagnosis (web-based viewers, see Fig. 5).

phase shifts, the phase shift of the field is retrieved at each point in the field of view.

Critical to the operation of the tissue scanner is the ability to perform phase-shifting and computation in real time. To this end, we developed an acquisition software that seamlessly combines CPU and GPU processing [Fig. 1(b)]. The SLIM phase retrieval computation occurs on a separate thread while the microscope stage moves to the next position (see Sec. 2).
Computation is performed on the GPU, freeing the CPU for data serialization tasks, such as reading the camera buffer or writing files to the disk. To account for sample variation, simplify the operating procedure, and reduce variation between acquisitions, a depth scan optimizes the acquired focus at each tile with the final "in-focus" image acquired at an interpolated z-position. We address the global tilt (due to stage, sample, mounting) by specifying focus points and performing the depth 
(a)
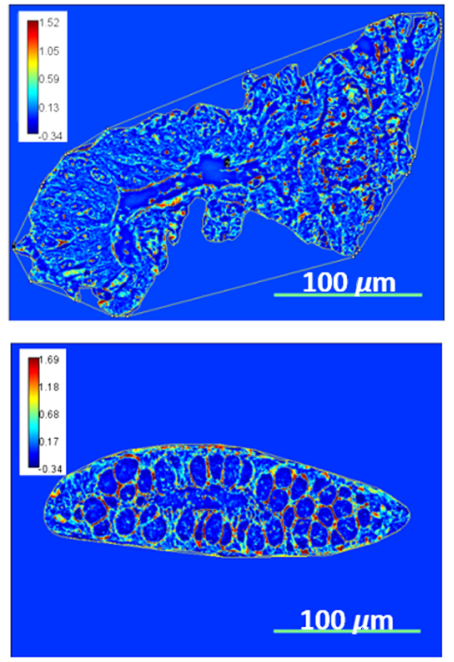

(b)
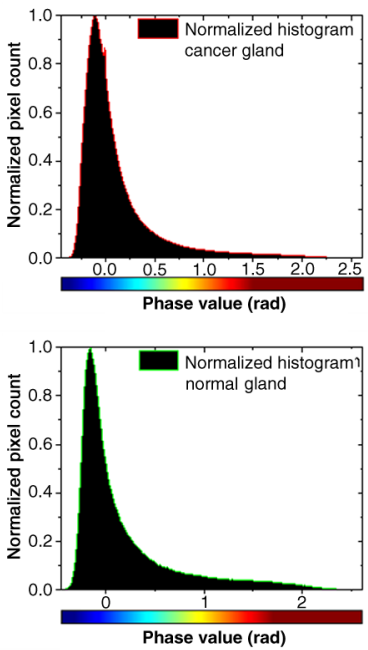

(c)

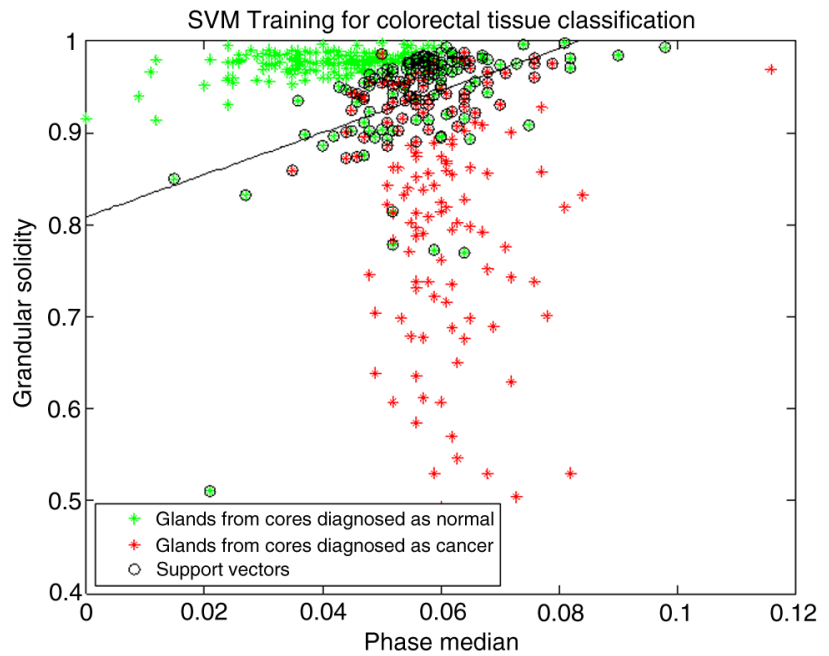

Fig. 8 Quantitative parameters for classification. Our classification method augments phase information with the geometric structure of the gland. Scanned images are manually segmented into glands, whose solidity ("glandular solidity") and median phase value are used to train the SVM-based classifier used in this work. (a) Gland identification, (b) feature extraction, and (c) classification.

scan offset from this tilt, drastically reducing the scan volume. These five or six manual focus points are the same points used to locate the sample. In the future, we plan to identify the scan area by performing a "prescan" at a low magnification $(2 \times)$. The final SLIM images [Fig. 1(c)] are displayed at up to 15 frames per second, as limited by the SLM refresh rate-which has shown improvement since the start of this work. ${ }^{57}$

Scanning large fields of view, e.g., entire microscope slides, and assembling the resulting images into single files required the development of new dedicated software tools [Fig. 7(a)]. The resulting phase images are prepared for archival storage with an image alignment and rasterization code developed inhouse (Sec. 2.5). The local overlap between neighboring tiles is estimated with a parallel Python code, where disk access is masked by computation [Fig. 7(b)]. Specifically, a "producer" thread reads images from the disk while a second "consumer" thread performs phase correlation and peak detection. Discrepancies between the locations of tile pairs are resolved through global optimization, following a least-squares model [Fig. 7(c)]. After background correction, to account for alignment artifacts, the overlapping tiles are combined into bitmaps suitable for display. This process reduces $\sim 200$ gigabytes of floating point TIF
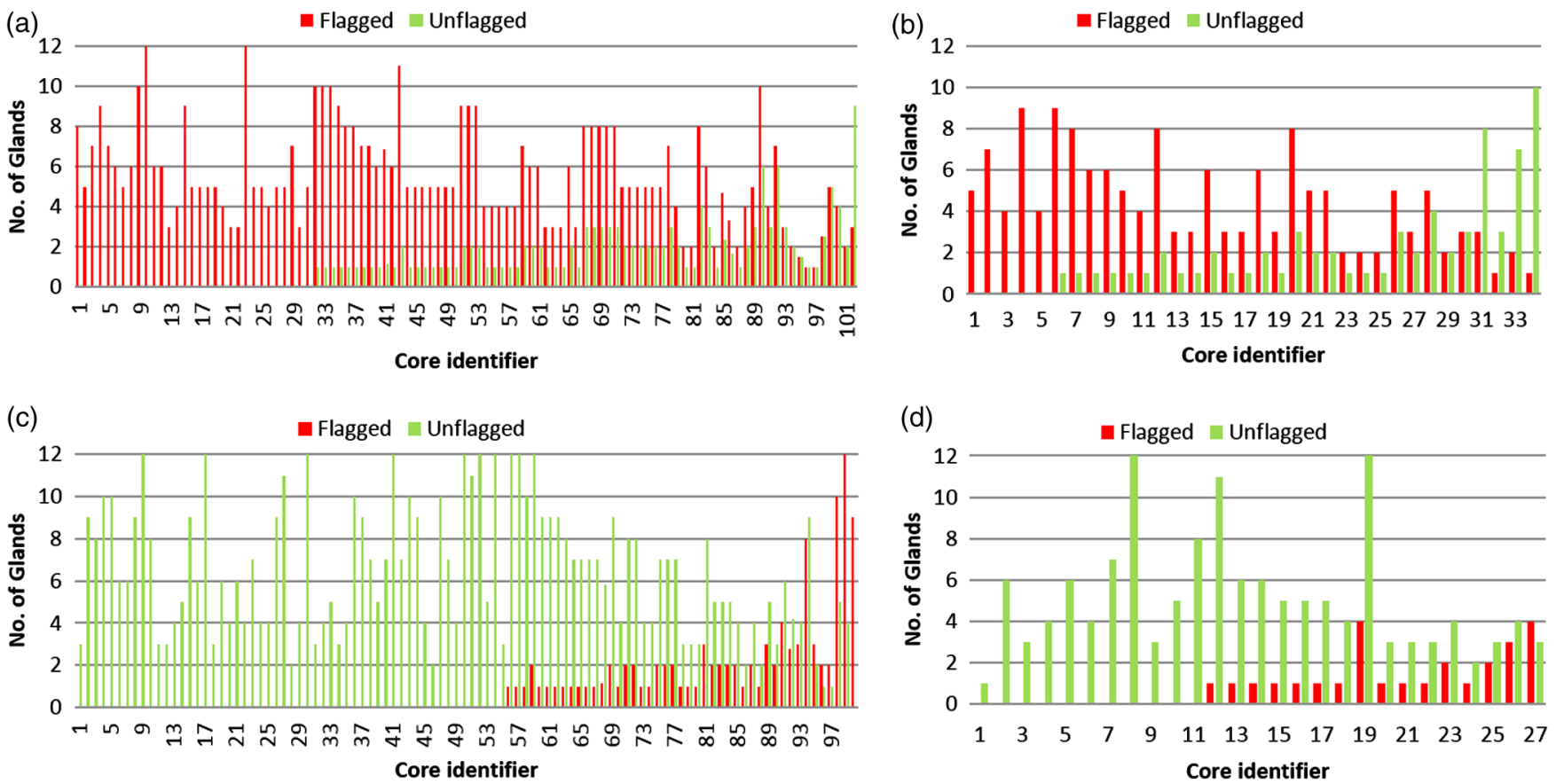

Fig. 9 Tissue classification using an SVM. Screening of cores for each of the four groups: (a) cancer, (b) dysplastic, (c) normal, and (d) hyperplastic. A typical core contains several glands, each being evaluated using the SVM classifier. The consensus of these evaluations is used to diagnosis the core. 
data into 2 gigabytes of web-ready JPEGs. The final tiled images are stored and available for access remotely, through "deep zoom" style image viewers, such as Google Maps" ${ }^{\mathrm{TM}}$ [Fig. 7(f)].

\subsection{Quantitative Classification of Tissue Microarray Cores}

We used the tissue scanner to image a TMA consisting of 0.6$\mathrm{mm}$ diameter colon core duplicates of tumor, normal mucosa, dysplastic mucosa, and hyperplastic mucosa from 131 patients who underwent colon resection. To quantitatively classify tissue into the categories of high index of suspicion (dysplasia, intramucosal carcinoma, and carcinoma) and low index of suspicion (normal and hyperplasia), an SVM classifier was built using glandular solidity and glandular phase median as the support vectors (see Sec. 2 for details).
Figure 8 shows the procedure for tissue classification. In short, cancer is detected through these two parameters. We use SVM to determine their optimal fusion, which in the subsequent text is referred to as the "index of suspicion." ${ }^{8}$ We note that while individually, the morphological and quantitative features are insufficient for clinical discrimination, when combined together, they provide a strong diagnostic marker not available through conventional, label-based imaging techniques.

The SVM classifier was first tested on three TMA slides, consisting of 816 glands from 100 cores designated as normal, 168 glands from 27 cores designated as hyperplastic, 219 glands from 34 cores designated as dysplastic, and 704 glands from 102 cores designated as cancerous. The distribution of gland types across the cores is shown in Fig. 9. Using our analysis based on gland solidity and phase values, $86 \%$ of the glands from normal cores and $85 \%$ of the glands from hyperplastic cores were

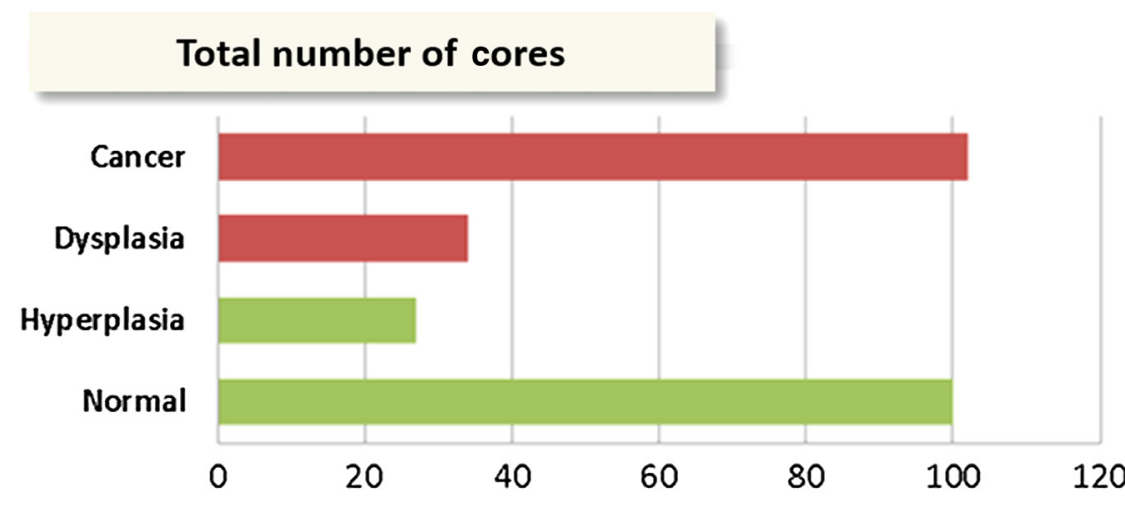

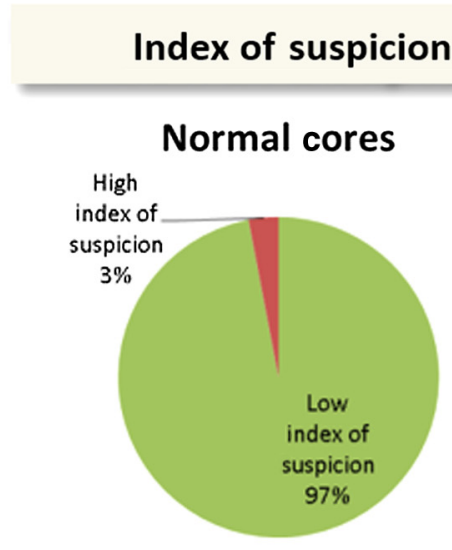

Dysplastic cores

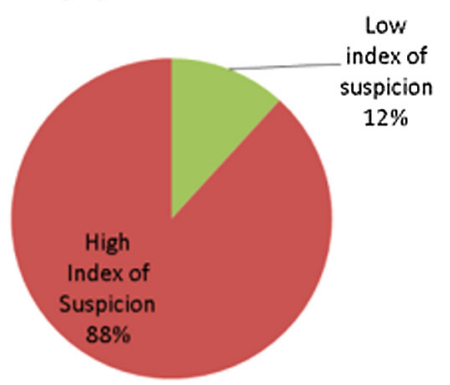

Hyperplastic cores

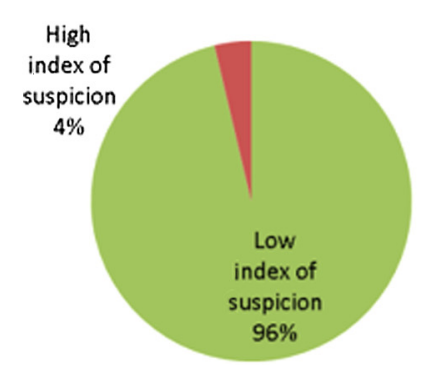

Cancerous cores

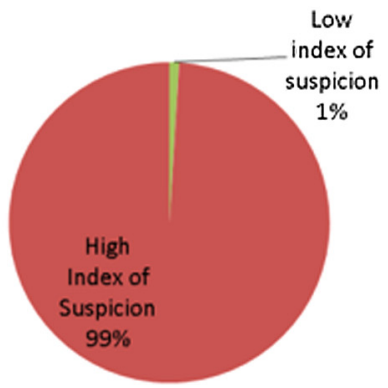

Fig. 10 Classification accuracy of colorectal tissue. The accuracy of classification of normal, hyperplastic, dysplastic, and cancerous cores into the "high index of suspicion" and "low index of suspicion" groups is shown. The high index of suspicion (dysplasia, cancer) cases are flagged with $96.3 \%$ sensitivity and $95.3 \%$ specificity. 
classified into the low index of suspicion group. Furthermore, $68 \%$ of the glands from dysplastic cores and $80 \%$ of the glands from cancerous cores were classified into the high index of suspicion group. Figure 10 summarizes these results.

It should be noted that, for a core to be diagnosed as normal or hyperplastic, all glands in that core must show normal or hyperplastic morphology on the H\&E images. However, for a core to be diagnosed with dysplasia, intramucosal carcinoma, or carcinoma, a minimum of one gland has to display this morphology on the H\&E image, and other glands could have normal or hyperplastic morphology. This explains the higher discrimination accuracy seen in glandular classification from cases in the low index of suspicion group, in comparison with cases in the high index of suspicion group. Figure 10 shows the number of glands classified as high index of suspicion and low index of suspicion in each core belonging to the normal, hyperplasia, dysplasia, and carcinoma categories.

Our end goal is to provide a quantitative screening tool, i.e., to rule out normal cases and flag cores with suspected cancer or dysplasia for further examination by a pathologist. To this end, the risk stratification of multiple glands from each core was pooled together and a threshold was set as follows: if 50\% or more glands from a single core were classified as "high index of suspicion," then the core was flagged. This resulted in 135 cores being flagged for further analysis by the pathologist. A closer examination of the flagged cores showed that $88 \%$ of all cores with dysplasia and $99 \%$ of all cores with cancer were flagged (Fig. 10). Additionally, 5\% of all normal cores and $4 \%$ of all hyperplastic cores were incorrectly flagged as high index of suspicion. The overall sensitivity and specificity for identification of high index of suspicion cases were $96.3 \%$ and $96.9 \%$, respectively.

\subsection{Quantitative Classification of Full Tissue Slide}

To illustrate the operation of our imaging and classification analysis on full biopsies, a surgically resected colon sample was imaged using the SLIM tissue scanner (see Sec. 2 for details). The large tissue image was cropped to $10,000 \times$ 10,000 pixel regions over which the glands were segmented for extracting the phase median and glandular solidity values. The SVM classifier flagged the glands as either high or low index of suspicion. As discussed in Sec. 2.10, the variation in tissue thickness (if any) corresponds to morphological changes. As a control, we used an adjacent H\&E section with the pathologist's diagnosis to compare against our result. As shown in Fig. 11, the quantitative classifier flagged all the regions with cancerous or dysplastic regions as high index of suspicion, and all benign regions were successfully assigned a low index of suspicion.

\section{Discussion}

Our results show that SLIM data have diagnostic value and that high and low index of suspicion cases can be classified extremely well. Selecting the dysplasia and cancer cases with $96 \%$ sensitivity and $95 \%$ specificity is very promising. As shown with previous reports of label-free imaging for diagnosis, ${ }^{19,59-70}$ we do not attempt to replace the clinical workflow but to complement it with valuable information that results in higher accuracy, reduced manual time, and increased throughput. Histopathology following colonoscopy tissue resection continues to remain the gold-standard for diagnosis of colorectal cancer and dysplasia. At the same time, we believe that, if valuable, a new technology can and will be incorporated into the existing process. The semiautomated pap smear analysis is a good example where the existing workflow was adjusted to incorporate a new tool that brings value. Stains are continuously developed as well and can be regarded as a disruption of the common workflow, but, because they significantly boost specificity, in some cases, this disruption is worthwhile. In our case, we envision a process in which SLIM imaging is performed before staining, possible in conjunction with $\mathrm{H} \& \mathrm{E}$. Alternatively, SLIM can be performed on successive slices, as is the

\section{Surgically resected colon}

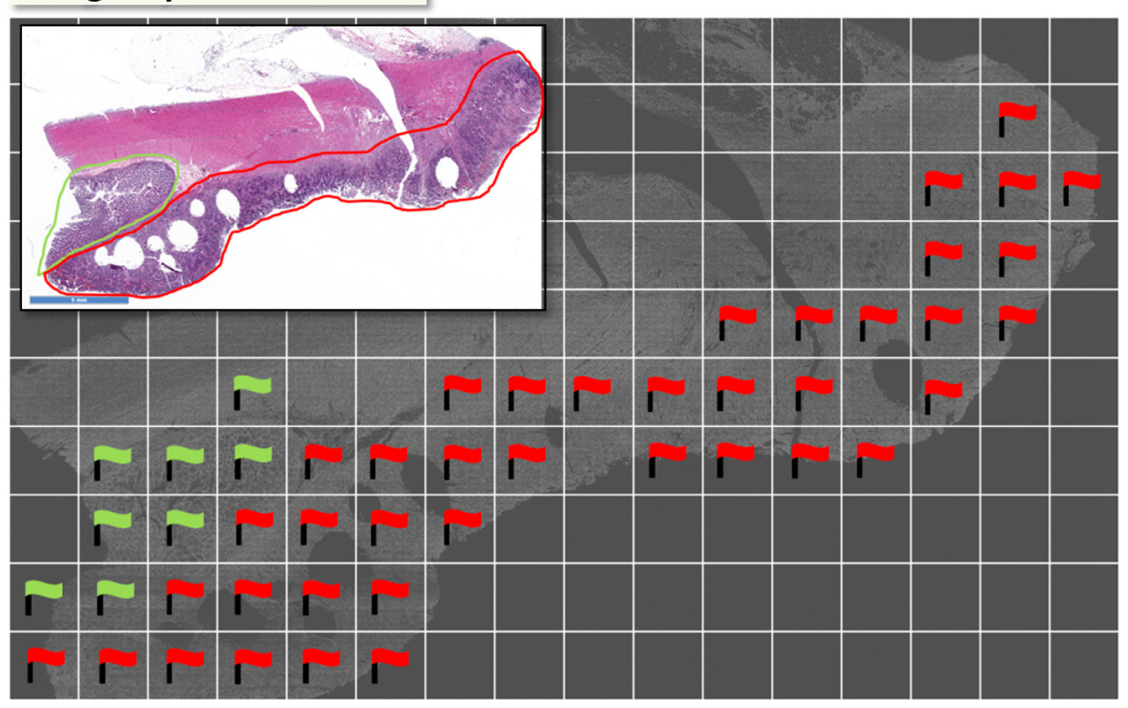

Fig. 11 Biopsy flagged with regions of "high" (red) and "low"' suspicion (green). After assembly, the image was analyzed in chunks of $10,000 \times 10,000$ pixel regions with glands in each region evaluated according to the SVM model. The consensus of constituent glands is represented with a "green" or "red" flag indicating low or high index of suspicion, respectively. Inset: H\&E stained parallel section, showing the red-bordered tumor and green-border benign region, as indicated by the pathologist. 
case with additional staining, and, as shown here, this can now be accomplished without significant disruption.

Due to its common-path interferometric geometry, SLIM is extremely stable and, thus, provides information about tissue architecture at the nanoscale. This type of data is specific to interferometry. An appealing feature of this system is that it can be integrated with existing microscopes with which the pathologist is already familiar. In our approach, a parallel $\mathrm{C}+$ + code drives the hardware, achieving optimal performance with half the time spent moving the stage and half acquiring the interferometric image. Crucial to the performance of the code is our use of references to memory locations (pointers) to avoid redundant data copies. As a result, we achieved an order of magnitude increase in throughput compared with serial systems, creating a compromise-free imaging system, which allowed us to establish a new quantitative marker for colorectal cancer screening.

SLIM has a high sensitivity and high specificity for flagging cases requiring pathological examination, and it flags specific regions on large tissue samples, such as biopsies, requiring the attention of pathologists. Therefore, SLIM can help optimize diagnostic workflow in colorectal screening programs. This demonstration, along with the ability to effortlessly generated and share large datasets, will serve as motivation for exploring new disease markers and developing automatic diagnostic algorithms.

\section{Disclosures}

G.P. has a financial interest in Phi Optics, Inc., a company that commercializes quantitative phase imaging technology for materials and life science applications.

\section{Acknowledgments}

We would like to thank Dr. Catalin Chiritescu for helping to maintain the instrument used in this work. This work was supported by the National Science Foundation (CBET-1040461 and IIP-1353368) and Agilent Laboratories. For more information, visit light.ece.illinois.edu.

\section{References}

1. T. Muto, H. J. Bussey, and B. C. Morson, "The evolution of cancer of the colon and rectum," Cancer 36(6), 2251-2270 (1975).

2. N. Howlader et al., SEER Cancer Statistics Review, 1975-2011, N. Howlader et al. Eds., National Cancer Institute, Bethesda, Maryland (2014).

3. Centers for Disease Control and Prevention (CDC), "Vital signs: colorectal cancer screening test use-United States, 2012," Morb. Mortal. Wkly. Rep. 62(44), 881-888 (2013).

4. S. J. Winawer, "Natural history of colorectal cancer," Am. J. Med. 106(1A), 3S-6S (1999).

5. A. Giacosa, F. Frascio, and F. Munizzi, "Epidemiology of colorectal polyps," Tech. Coloproctol. 8(Suppl. 2), s243-247 (2004).

6. K. Pollitz et al., Coverage of Colonoscopies Under the Affordable Care Act's Prevention Benefit, The Kaiser Family Foundation, American Cancer Society, National Colorectal Cancer Roundtable, Menlo Park, California (2012).

7. "Colonoscopy Coverage: sample billing guidelines," Blue Cross Blue Shield Association.

8. J. J. Sung et al., "Increasing incidence of colorectal cancer in Asia: implications for screening,' Lancet Oncol. 6(11), 871-876 (2005).

9. W. K. Leung et al., "Colorectal neoplasia in Asia: a multicenter colonoscopy survey in symptomatic patients," Gastrointest. Endoscopy 64(5), 751-759 (2006).

10. T. Konishi et al., "Difference in incidence of colorectal cancer between men and women in Asia," Lancet Oncol. 7(2), 104-105 (2006).
11. L. Pantanowitz, "Automated pap tests," in Practical Informatics for Cytopathology, L. Pantanowitz and A. V. Parwani, Eds., pp. 147-155, Springer, New York (2014).

12. Y. C. Chen et al., "X-ray computed tomography of holographically fabricated three-dimensional photonic crystals," Adv. Mater. 24(21), 2863-2868 (2012).

13. "Quality assurance on the BD focalpoint slide profiler," in $B D$ FocalPoint $^{\mathrm{TM}}$ GS Imaging System: Intelligent Pap Imaging ${ }^{\mathrm{TM}}$, pp. 19-14, Becton Dickinson, Franklin Lakes, New Jersey.

14. V. Backman et al., "Detection of preinvasive cancer cells," Nature 406(6791), 35-36 (2000).

15. D. C. Fernandez et al., "Infrared spectroscopic imaging for histopathologic recognition," Nat. Biotechnol. 23(4), 469-474 (2005).

16. F. E. Robles et al., "Detection of early colorectal cancer development in the azoxymethane rat carcinogenesis model with Fourier domain low coherence interferometry," Biomed. Opt. Express 1(2), 736-745 (2010).

17. B. J. Vakoc et al., "Cancer imaging by optical coherence tomography: preclinical progress and clinical potential," Nat. Rev. Cancer 12(5), 363-368 (2012).

18. A. Greenbaum et al., "Wide-field computational imaging of pathology slides using lens-free on-chip microscopy," Sci. Trans. Med. 6(267), 267ra175 (2014).

19. S. Uttam et al., "Early prediction of cancer progression by depth-resolved nanoscale mapping of nuclear architecture from unstained tissue specimens," Cancer Res. 75(22), 4718-4727 (2015).

20. R. Horstmeyer et al., "Digital pathology with Fourier ptychography," Comput. Med. Imaging Graphics 42, 38-43 (2015).

21. R. A. McLaughlin et al., "Parametric imaging of cancer with optical coherence tomography," J. Biomed. Opt. 15(4), 046029 (2010).

22. N. Iftimia et al., "Fluorescence-guided optical coherence tomography imaging for colon cancer screening: a preliminary mouse study," Biomed. Opt. Express 3(1), 178-191 (2012).

23. K. Grieve et al., "A feasibility study of full-field optical coherence tomography for rapid evaluation of EUS-guided microbiopsy specimens," Gastrointest. Endoscopy 81(2), 342-350 (2015).

24. V. Backman and H. K. Roy, "Advances in biophotonics detection of field carcinogenesis for colon cancer risk stratification," J. Cancer 4(3), 251-261 (2013).

25. M. Lee et al., "Label-free optical quantification of structural alterations in Alzheimer's disease," Sci. Rep. 6, 31034 (2016).

26. G. Popescu, "Quantitative Phase Imaging of Cells and Tissues" (2011).

27. G. A. Dunn and D. Zicha, "Using DRIMAPS system of transmission interference microscopy to study cell behavior," in Cell Biology: a Laboratory Handbook, 2nd ed., Academic Press, New York (1997).

28. M. Mir et al., "Optical measurement of cycle-dependent cell growth," Proc. Natl. Acad. Sci. U. S. A. 108(32), 13124-13129 (2011).

29. M. Mir et al., "Highly sensitive quantitative imaging for monitoring single cancer cell growth kinetics and drug response," PLoS One 9(2), e89000 (2014).

30. G. Popescu et al., "Optical measurement of cell membrane tension," Phys. Rev. Lett. 97(21), 218101 (2006).

31. R. Wang et al., "Dispersion-relation phase spectroscopy of intracellular transport," Opt. Express 19(21), 20571-20579 (2011).

32. W. Ru et al., "One-dimensional deterministic transport in neurons measured by dispersion-relation phase spectroscopy," J. Phys. Condens. Matter 23(37), 374107 (2011).

33. S. Ceballos et al., "Active intracellular transport in metastatic cells studied by spatial light interference microscopy," J. Biomed. Opt. 20(11), 111209 (2015).

34. M. E. Kandel et al., "Label-free imaging of single microtubule dynamics using spatial light interference microscopy," ACS Nano 11(1), 647-655 (2017).

35. F. Charriere et al., "Cell refractive index tomography by digital holographic microscopy," Opt. Lett. 31(2), 178-180 (2006).

36. W. Choi et al., "Tomographic phase microscopy," Nat. Methods 4(9), 717-719 (2007).

37. Z. Wang et al., "Spatial light interference tomography (SLIT)," Opt. Express 19(21), 19907-19918 (2011).

38. Y. Cotte et al., "Marker-free phase nanoscopy," Nat. Photonics 7(2), 113-117 (2013).

39. T. Kim et al., "White-light diffraction tomography of unlabelled live cells," Nat. Photonics 8(3), 256-263 (2014). 
40. Y. Park et al., "Measurement of red blood cell mechanics during morphological changes," Proc. Natl. Acad. Sci. U. S. A. 107(15), 67316736 (2010).

41. Y. K. Park et al., "Metabolic remodeling of the human red blood cell membrane," Proc. Natl. Acad. Sci. 107, 1289 (2010).

42. L. Miccio et al., "Red blood cell as an adaptive optofluidic microlens," Nat. Commun. 6, 6502 (2015).

43. H. Park et al., "Characterizations of individual mouse red blood cells parasitized by Babesia microti using 3-D holographic microscopy," Sci. Rep. 5, 10827 (2015).

44. Z. Wang et al., "Tissue refractive index as marker of disease," J. Biomed. Opt. 16(11), 116017 (2011).

45. H. Ding et al., "Measuring the scattering parameters of tissues from quantitative phase imaging of thin slices," Opt. Lett. 36(12), 22812283 (2011).

46. S. Sridharan et al., "Prediction of prostate cancer recurrence using quantitative phase imaging," Sci. Rep. 5, 9976 (2015).

47. S. Sridharan et al., "Prediction of prostate cancer recurrence using quantitative phase imaging: validation on a general population," Sci. Rep. 6, 33818 (2016).

48. Z. Wang et al., "Spatial light interference microscopy (SLIM)," Opt. Express 19(2), 1016-1026 (2011).

49. N. M. Master et al., "Performance analysis of commodity and enterprise class flash devices," in 5th Petascale Data Storage Workshop (PDSW), pp. 1-5 (2010).

50. P. Alliez and A. Fabri, "CGAL: the computational geometry algorithms library," in ACM SIGGRAPH 2016 Courses, pp. 1-8, ACM, Anaheim, California (2016).

51. L. Firestone et al., "Comparison of autofocus methods for automated microscopy," Cytometry 12(3), 195-206 (1991).

52. W. H. Press et al., Numerical Recipes 3rd Edition: The Art of Scientific Computing, Cambridge University Press, New York (2007).

53. E. D. Castro and C. Morandi, "Registration of translated and rotated images using finite Fourier transforms," IEEE Trans. Pattern Anal. Mach. Intell. PAMI-9(5), 700-703 (1987).

54. S. Saalfeld et al., "CATMAID: collaborative annotation toolkit for massive amounts of image data," Bioinformatics 25(15), 1984-1986 (2009).

55. J. MacQueen, "Some methods for classification and analysis of multivariate observations," in Fifth Berkeley Symp. on Mathematical Statistics and Probability, Proc. of the Fifth Berkeley Symp. on Mathematical Statistics and Probability, Volume 1: Statistics, pp. 281-297 (1967).

56. J. Kononen et al., "Tissue microarrays for high-throughput molecular profiling of tumor specimens," Nat. Med. 4(7), 844-847 (1998).

57. G. Thalhammer et al., "Speeding up liquid crystal SLMs using overdrive with phase change reduction," Opt. Express 21(2), 1779-1797 (2013).

58. B. Berwick, "An idiot's guide to support vector machiens (SVMs)," in 6.034 Artificial Intelligence-Recitations, MIT (2011).

59. J. J. Casciari, S. V. Sotirchos, and R. M. Sutherland, "Glucose diffusivity in multicellular tumor spheroids," Cancer Res. 48(14), 3905-3909 (1988).

60. H. K. Roy et al., "Association between rectal optical signatures and colonic neoplasia: potential applications for screening," Cancer Res. 69(10), 4476-4483 (2009).

61. E. I. Galanzha et al., "In vivo, noninvasive, label-free detection and eradication of circulating metastatic melanoma cells using two-color photoacoustic flow cytometry with a diode laser," Cancer Res. 69(20), 7926-7934 (2009).

62. H. Subramanian et al., "Nanoscale cellular changes in field carcinogenesis detected by partial wave spectroscopy," Cancer Res. 69(13), 53575363 (2009).

63. T. W. Remmerbach et al., "Oral cancer diagnosis by mechanical phenotyping," Cancer Res. 69(5), 1728-1732 (2009).

64. H. K. Roy et al., "Optical detection of buccal epithelial nanoarchitectural alterations in patients harboring lung cancer: implications for screening," Cancer Res. 70(20), 7748-7754 (2010).

65. D. Damania et al., "Nanocytology of rectal colonocytes to assess risk of colon cancer based on field cancerization," Cancer Res. 72(11), 27202727 (2012).

66. H. G. Jahnke et al., "Direct chemosensitivity monitoring ex vivo on undissociated melanoma tumor tissue by impedance spectroscopy," Cancer Res. 74(22), 6408-6418 (2014).
67. S. J. Erickson-Bhatt et al., "Real-time imaging of the resection bed using a handheld probe to reduce incidence of microscopic positive margins in cancer surgery," Cancer Res. 75(18), 3706-3712 (2015).

68. A. Gamez-Pozo et al., "Combined label-free quantitative proteomics and microRNA expression analysis of breast cancer unravel molecular differences with clinical implications," Cancer Res. 75(11), 2243-2253 (2015).

69. F. K. Lu et al., "Label-free neurosurgical pathology with stimulated Raman imaging," Cancer Res. 76(12), 3451-3462 (2016).

70. R. Pal et al., "Remodeling of the epithelial-connective tissue interface in oral epithelial dysplasia as visualized by noninvasive 3D imaging," Cancer Res. 76(16), 4637-4647 (2016).

Mikhail E. Kandel is a graduate student working on computational tools and instrumentation for phase-shifting interferometers. His research interests include studying image formation to improve tomography, time-lapse microscopy to monitor adherent cell populations, and developing new markers to remove observer bias in pathology.

Shamira Sridharan received her PhD in Bioengineering with a focus on applications of quantitative phase imaging in genitourinary and gastro-intestinal pathology, from the University of Illinois at UrbanaChampaign. She is currently a clinical research postdoctoral fellow at the University of California, Davis and she works on clinical applications of multimodal imaging systems.

Jon Liang graduated from the University of Illinois at UrbanaChampaign with a bachelor's degree in Engineering Physics. During his time at Illinois he worked with Dr. Shamira Sridharan and Dr. Gabriel Popescu in the QLI Lab on projects for the application of SLIM imaging on cancer biopsies. He currently works at Epic Systems in Madison Wisconsin as an Integration Engineer.

Zelun Luo is a graduate student in computer science at Stanford University. He is currently working in the Stanford Vision Lab with Professor Fei-Fei Li. Before joining Stanford, he finished his bachelor's degree in computer engineering at the University of Illinois at Urbana-Champaign, during which he worked in the QLI Laboratory with Professor Gabriel Popescu, developing phase imaging platform for tissue microarray scans. His interests include computer vision and Al-assisted healthcare.

Virgilia Macias is a research pathologist with background in oncologic surgical pathology, training in electron microscopy and immunohistochemistry. She did her training in Mexico. She is currently a research assistant professor in the Transdisciplinary Pathology Department at the University of Illinois at Chicago. Her research interest is mainly focused on investigation and/or validation of prostate cancer biomarkers. She is involved in laser microdissection, quantitative image analysis, tissue microarray (TMA) construction, and tissue banking.

Roshan Patel is a board-certified Pathologist in both Anatomic and Clinical Pathology, and is fellowship trained in Gastrointestinal and Liver Pathology. He completed medical school at Rush Medical College and a residency at the University of Illinois Medical Center, both in Chicago. He works hand-in-hand with physicians to identify and evaluate disease. The health and well-being of patients is most important to him.

Andre Kajdacsy-Balla is a professor and director of transdisciplinary pathology at the University of Illinois at Chicago. His clinical focus is anatomic pathology with special interest in gynecologic pathology and prostate cancer. His research interests are in the areas of tissue banking, TMAs, application of molecular techniques to tissue pathology, prostate cancer clinical outcomes prediction methods, and the effect of environmental agents on prostate cancer progression and metastasis.

Grace Guzman is an associate professor of Pathology at the University of Illinois Hospital and Health Sciences System where she teaches liver and gastrointestinal pathology and serves as the medical director of liver pathology services. She focuses on the translational study of liver diseases, hepatocellular and colon carcinoma, 
addressing pathogenesis, cell signaling, development of human tissue arrays, and characterizing biomarkers.

Gabriel Popescu received his $\mathrm{PhD}$ in Optics from the School of Optics/CREOL (now the College of Optics and Photonics), UCF in 2002. He continued with Michael Feld at Massachusetts Institute of Technology as a postdoctoral associate. In 2007, he joined ECE and the Beckman Institute at UIUC, where he has been active in QPI, on which he authored a book (McGraw-Hill, 2011). He founded Phi Optics, a start-up company that commercializes QPI technology. $\mathrm{He}$ is an OSA and SPIE fellow.

Biographies for the other authors are not available. 